\title{
Comparison of the clinical features and outcomes of infective endocarditis between hemodialysis and non-hemodialysis patients
}

This article was published in the following Dove Press journal:

Therapeutics and Clinical Risk Management

24 May 2017

Number of times this article has been viewed

\author{
Ching-Chung Hsiao',* \\ Cheng-Hao Weng ${ }^{1,2, *}$ \\ Yi-Jung $\mathrm{Li}^{1}$ \\ $\mathrm{Hsin}-\mathrm{Hsu} \mathrm{Wu}^{1,2}$ \\ Yung-Chang Chen' \\ Yu-Ming Chen' \\ Hsiang-Hao Hsu' \\ Ya-Chung Tian'
}

'Kidney Research Center and Department of Nephrology, Chang Gung Memorial Hospital, Taipei, ${ }^{2}$ Graduate Institute of Clinical Medical Sciences, Chang Gung University,

Taoyuan, Taiwan

*These authors contributed equally to this work
Correspondence: Ya-Chung Tian Department of Nephrology, Chang Gung Memorial Hospital, 199 Tun-Hwa North Road, Taipei 105, Taiwan

Tel +8863328 I200 (ext 8I8I)

Fax +88633282173

Email dryctian@cgmh.org.tw
Abstract: Hemodialysis (HD) patients are more susceptible to infective endocarditis (IE) due to the increased risk of bacterial invasion through intravascular access. However, it remains unclear whether the causative organisms and outcomes of IE in HD patients differ from those in non-HD patients. This study clarified the differences in clinical presentation and outcomes between HD and non-HD patients. At our hospital, we performed a retrospective study of $39 \mathrm{HD}$ and 51 non-HD patients with echocardiography-confirmed IE between June 2000 and February 2007. No differences in sex, intravenous drug use, previous diagnosis of congestive heart failure, and previous valvular surgery were observed between these two groups. The number of patients with diabetic mellitus in these two groups was significantly different (28.2\% HD vs $5.9 \%$ non-HD patients). The C-reactive protein levels in the two groups were not significantly different. By contrast, the erythrocyte sedimentation rate was significantly higher in the HD patients (HD vs non-HD: $87.2 \pm 33.32$ vs $52.96 \pm 28.19$ ). The incidence of IE involving the mitral valve (MV; 45.1\%) or the aortic valve (AV; 43.1\%) was similar among the non-HD patients, whereas a preference of IE involving the MV (79.5\%) over the AV (15.4\%) was noted among the HD patients. The HD patients had a significantly higher Staphylococcus aureus infection rate (HD: $46.2 \%$; non-HD: 27.5\%). The proportion of methicillin-resistant S. aureus (MRSA; $83.8 \%$ ) infection accounting for S. aureus IE in the HD group was higher than that $(28.6 \%)$ in the non-HD group. The in-hospital mortality rate did not differ between the two groups. In conclusion, compared with non-HD patients, a propensity of IE involving the MV and a higher MRSA infection rate were observed in HD patients. The in-hospital mortality rate of echocardiography-confirmed IE did not differ between the two groups.

Keywords: infective endocarditis, hemodialysis, mitral valve, aortic valve, Staphylococcus aureus

\section{Introduction}

Over the past two decades, the incidence of infective endocarditis (IE) has remained unchanged despite improvements in medications and health care. ${ }^{1,2}$ Rheumatic heart disease with damaged heart valves was the major cause of IE in the pre-antibiotic era. ${ }^{3}$ However, the main causes of IE have changed to intravenous (IV) drug use and intravascular prosthesis use. Compared with the general population, hemodialysis (HD) patients requiring repeated arteriovenous fistula punctures for HD are at a higher risk of IE. The incidence of community-acquired native valve IE ranges from 1.7 to 4.45 episodes per 100,000 person-years, but the incidence of IE complicating bacteremic episodes in HD patients can be up to $12 \% .{ }^{1-6}$ Strom et al reported that the relative risk of IE in HD patients was as much as 16.9 times that of IE in the general population. ${ }^{7}$ Moreover, non-HD 
patients have a $90 \%-95 \%$ cure rate, whereas in HD patients with IE, the mortality rate ranges from $21.6 \%$ to $50 \% .^{8-14}$

Several possible risk factors, including a higher incidence of congestive heart failure (CHF); the calcification of heart valves, which frequently causes damage to the valves; a higher rate of exposure to bacteremia due to repetitive vascular puncture; and more invasive causative micro-organisms such as Staphylococcus aureus, contribute to the poorer outcomes of IE in HD patients than in non-HD patients. ${ }^{15-17}$ All of these factors also make HD patients more susceptible to IE and result in different manifestations of IE in HD patients (such as different valve involvement and species of micro-organisms) relative to non-HD patients. Nevertheless, the differences between IE in HD and non-HD patients have not been directly compared. The aim of this study was to concurrently compare the contributing factors and outcomes of IE in HD and non-HD patients.

\section{Methods}

\section{Study population}

The study was approved by the institutional review board of Chang Gung Medical Foundation, and written informed consent from each participant was waived by the institutional review board of Chang Gung Medical Foundation as personal information have been anonymized and deidentified for this study. The computerized data of patients with a discharge diagnosis of IE confirmed through echocardiography were retrospectively analyzed at Chang Gung Memorial Hospital, a tertiary teaching hospital, from June 2000 to February 2007. The modified Duke criteria were adopted for screening the diagnosis of IE. Only patients with a diagnosis of IE, confirmed based on the findings of vegetations on the cardiac valve detected through either transesophageal or transthoracic echocardiography, were enrolled in this study. Demographic, clinical, and laboratory data were obtained for each patient, including sex, age, underlying comorbidities, erythrocyte sedimentation rate (ESR), levels of C-reactive protein (CRP) and hematocrit, type of infectious micro-organisms, valvular location of vegetations, history of previous valvular replacement, surgical repair of the damaged valves, hospital mortality rate, and period from the diagnosis of IE to death.

\section{Statistical analysis}

Continuous variables are expressed as mean \pm standard deviation, with comparisons performed using Student's $t$-test or one-way analysis of variance (ANOVA). Univariate analysis was performed using the Pearson chi-square test for categorical variables. A two-sided Fisher's exact test was used if the expected count in any cell was $<5$. Statistical significance was established if the $P$-value was $<0.05$.

\section{Results}

Ninety patients with a diagnosis of IE confirmed through echocardiography were enrolled in the current study from June 2000 to February 2007. Among them, 39 patients were receiving maintenance HD when IE was confirmed, and the remaining 51 patients were classified as the non-HD group. The HD group comprised 19 male and 20 female patients, and the non-HD group comprised 32 male and 19 female patients (Table 1). The average ages of the HD and non-HD patients were $59.46 \pm 14.70$ and $52.44 \pm 17.72$ years, respectively. No differences were observed in the distributions of sex and age between the two groups. The number of patients with diabetic mellitus significantly differed between both the groups (28.2\% HD vs $5.9 \%$ non-HD patients; $P<0.05$ ). Because all the HD patients received repeated arteriovenous fistula punctures for dialysis, and only six non-HD patients (11.8\%) were IV drug abusers with a history of repetitive vascular punctures, a significant difference in the origin of IE through vascular access to the circulation was observed between these two groups $(100 \%$ of the HD patients with repeated vascular punctures vs $11.8 \%$ of the non-HD patients with a history of IV drug abuse; $P<0.01$ ). No significant difference was observed in the history of valvular surgery or the existence of CHF. The serum CRP levels were not significantly different between these two groups (HD vs non-HD: $138.27 \pm 81.01$ vs $155.68 \pm 124.46 ; P=0.281$ ). The ESR of the HD patients was significantly higher than that of the non-HD patients $(87.2 \pm 33.32$ vs $52.96 \pm 28.19 ; P=0.001)$. By contrast, the hematocrit level was significantly lower in the HD group (26.69 \pm 5.72 vs $30.33 \pm 6.25, P=0.016)$.

Table I Clinical characteristics of HD and non-HD patients with IE

\begin{tabular}{|c|c|c|c|}
\hline & $\begin{array}{l}\text { HD patients } \\
(n=39)\end{array}$ & $\begin{array}{l}\text { Non-HD patients } \\
(n=5 I)\end{array}$ & $P$-value \\
\hline Age (years) & $59.46 \pm 14.70$ & $52.44 \pm 17.72$ & 0.044 \\
\hline Sex (male/female) & $19 / 20$ & $32 / 19$ & NS \\
\hline Diabetes & II (28.2\%) & $3(5.9 \%)$ & 0.004 \\
\hline $\begin{array}{l}\text { Repetitive vascular } \\
\text { puncture }\end{array}$ & $39(100 \%)$ & $6(11.8 \%)$ & 0.027 \\
\hline Previous valve surgery & I (2.5\%) & $9(17 \%)$ & NS \\
\hline Congestive heart failure & II (28.2\%) & $22(43.1 \%)$ & NS \\
\hline Hematocrit & $26.69 \pm 5.72$ & $30.33 \pm 6.25$ & 0.016 \\
\hline $\mathrm{CRP}(\mathrm{mg} / \mathrm{dL})$ & $|38.27 \pm 8| .0 \mid$ & $|55.68 \pm| 24.46$ & NS \\
\hline ESR & $87.2 \pm 33.32$ & $52.96 \pm 28.19$ & 0.001 \\
\hline
\end{tabular}

Note: Data presented as mean \pm SD.

Abbreviations: CRP, C-reactive protein; ESR, erythrocyte sedimentation rate; HD, hemodialysis; IE, infective endocarditis; NS, non-significant; SD, standard deviation. 
Table 2 Valve involvement in HD and non-HD patients with IE

\begin{tabular}{llll}
\hline & $\begin{array}{l}\text { HD patients } \\
(\mathbf{n}=\mathbf{3 9})\end{array}$ & $\begin{array}{l}\text { Non-HD patients } \\
(\mathbf{n}=\mathbf{5} \mathbf{I})\end{array}$ & P-value \\
\hline Aortic valve & $6(15.4 \%)$ & $22(43.1 \%)$ & 0.004 \\
Mitral valve & $31(79.5 \%)$ & $23(45.1 \%)$ & \\
Tricuspid valve & $2(5.1 \%)$ & $6(11.8 \%)$ & \\
\hline
\end{tabular}

Abbreviations: $\mathrm{HD}$, hemodialysis; IE, infective endocarditis.

The valve involvement of IE detected through transthoracic echocardiography was markedly different in the HD and non-HD patients; vegetations were mostly discovered on the mitral valve (MV; 79.5\%) in the HD patients, whereas the majority of vegetations were evenly located on the aortic valve (AV; 43.1\%) or MV (45.1\%) in the non-HD patients (Table 2). In the current study, all the vegetations were discovered on a single valve, and no vegetation was simultaneously detected on other valves. Vegetations were seldom identified on the tricuspid valve (TV) in both groups.

Regarding the analysis of causative micro-organisms of IE, the species of bacteria isolated from blood cultures of the HD and non-HD patients were significantly different $(P<0.03)$. S. aureus was the main organism causing IE $(46.2 \%)$ in the HD patients, whereas other bacteria, including coagulase-negative Staphylococcus, Enterococcus, and Gram-negative species, were the most encountered organisms $(47.1 \%)$ in the non-HD patients with IE (Table 3). To clarify the response of $S$. aureus to methicillin in both the groups, $S$. aureus infection was further divided into methicillin-sensitive $S$. aureus (MSSA) and methicillinresistant S. aureus (MRSA) infection. The data demonstrated a significant difference in the proportion of MRSA-associated IE; MRSA-associated IE represented $83.3 \%$ of all the $S$. aureus-associated IE in the HD patients but only $28.6 \%$ of the $S$. aureus-associated IE in the non-HD patients $(P<0.05)$. The incidence of culture-negative IE was similar in both HD and non-HD patients.

Table 3 Causative bacterial species for IE in HD and non-HD patients

\begin{tabular}{llll}
\hline & $\begin{array}{l}\text { HD patients } \\
(\mathbf{n}=\mathbf{3 9})\end{array}$ & $\begin{array}{l}\text { Non-HD patients } \\
(\mathbf{n}=\mathbf{5} \text { I) }\end{array}$ & P-value \\
\hline Staphylococcus aureus & $18(46.2 \%)$ & $14(27.5 \%)$ & 0.03 \\
Others & $8(20.5 \%)$ & $24(47.1 \%)$ & \\
$\quad \begin{array}{l}\text { Coagulase-negative } \\
\text { Staphylococcus }\end{array}$ & 3 & 5 & \\
$\begin{array}{l}\text { Enterococcus } \\
\text { Gram-negative species }\end{array}$ & 2 & 3 & \\
$\quad$ Streptococcus species & 0 & 5 & \\
Culture-negative & $13(33.3 \%)$ & $13(25.5 \%)$ & \\
\hline
\end{tabular}

Abbreviations: HD, hemodialysis; IE, infective endocarditis.
Five HD patients (12.8\%) and 17 non-HD patients $(33.3 \%)$ received surgical treatment for active IE. Although more non-HD patients received surgery for IE, the difference did not reach statistical significance. Eighteen HD patients (46.2\%) with IE died in hospital, whereas 26 in-hospital deaths $(51 \%)$ were observed in the non-HD group. The in-hospital mortality rate in both groups was not different.

\section{Discussion}

The current retrospective study concurrently assessed the differences between IE in HD and non-HD patients of a tertiary teaching hospital. Our study is one of a few studies to compare clinical features and outcome of IE between HD and non-HD patients with similar backgrounds, which alleviates the confounding factors, such as variations in disease severity, diagnosis technique, and therapeutic modality, and thus, provides more reliable results.

The prognosis of IE is poor if diagnosis is delayed due to difficulty in discovering vegetations on cardiac valves. ${ }^{18}$ Definite or possible IE diagnosed based on the presence or absence of echocardiography-confirmed vegetations is the most decisive predictor of poor prognosis..$^{19}$ This study only enrolled patients with echocardiography-verified IE, resulting in a higher mortality rate in these patients compared with those reported in other studies. ${ }^{20,21}$

This study demonstrated that compared with the non-HD patients with IE, the HD patients with IE were older and had a higher incidence of diabetes. In addition, they had a higher frequency of vascular punctures, lower hematocrit levels, and higher ESRs. These differences in the clinical features of the HD and non-HD patients evaluated in this study probably only reflect the common characteristics of HD patients, because these patients were older and had higher incidence of diabetes and anemia, which resulted in higher ESRs.

Both CRP and ESR are markers of inflammation and infection. However, ESR is increased in different conditions, including pregnancy, anemia, autoimmune disorders, and some cancers. ${ }^{22}$ Although CRP is also elevated in late pregnancy, burns, cardiovascular diseases, and some cancers ${ }^{23} \mathrm{CRP}$ is more sensitive and accurate in reflecting the acute phase response to infection. Serum CRP levels are more useful than ESRs for predicting the response to antimicrobial therapy, complications, and prognosis. ${ }^{24-26}$ In our study, the serum CRP levels were elevated in all IE patients with or without HD. Unlike the significantly higher ESRs in the HD patients with IE in this study, the serum CRP levels were not different between the two groups. As many HD patients are anemic and thus have elevated ESR levels, 
ESR is not a good marker of IE in HD patients. Therefore, CRP has a higher diagnostic value for IE than does ESR in HD patients.

HD patients require frequent arteriovenous fistula punctures, leading to an increased exposure to bacteremia. Furthermore, the prevalence of CHF and valve calcification, both of which cause cardiac valve damage, is higher in HD patients. All these factors make HD patients more susceptible to IE and result in different manifestations of IE (such as differences in valve involvement and species of micro-organisms) in HD patients compared with non-HD patients.

The high prevalence of CHF and hyperparathyroidismassociated heart valve calcification frequently causes heart valve damage in HD patients. This study demonstrated significant differences in the type of heart valve involved in IE between the HD and non-HD patients. The most common heart valve involved in IE in the HD patients was the MV (81.5\%), followed by the AV (11.1\%) and TV (7.4\%). By contrast, no preference for MV involvement (47.2\%) over AV involvement (41.5\%) was observed in the non-HD patients. Several studies have reported that the most commonly affected heart valve in the HD patients with IE was the MV because this valve is more vulnerable to volume overload-related damage and calcifications than any other heart valves in HD patients. ${ }^{5,6,27}$ In line with the findings of these studies, our results also demonstrated that the MV was the most commonly involved heart valve in HD patients with IE. These findings indicate that vegetations on the MV should be carefully screened for during echocardiography in HD patients with suspicious IE.

HD patients require frequent arteriovenous fistula punctures or blood access through long-term implanted catheters, leading to an increased exposure to bacteremia from skin-colonizing bacteria that cause IE. Other than the entry of bacteria from vascular punctures, the non-HD patients exhibit a higher proportion of other routes for causing IE. For example, bacteria can enter the bloodstream through colorectal cancers and oral cancers or through urinary tract infections. These differences account for the variations in the species and virulence of causative micro-organisms in the HD and non-HD patients. In most studies, S. aureus has been reported as the most common causative organism for IE in non-HD patients, but other species of bacteria, such as oral and non-oral streptococci, also account for significant proportions of causative bacteria. ${ }^{28,29}$ Our study demonstrated that $S$. aureus accounted for $27 \%$ and $44 \%$ of the IE-causative bacteria in the non-HD and HD patients, respectively. Notably, MRSA accounted for $83.3 \%$ of $S$. aureus endocarditis in the HD patients, which was markedly higher than the $28.6 \%$ of $S$. aureus endocarditis caused by MRSA in the non-HD patients. This provides a therapeutic rationale for a possible change of antibiotics to vancomycin or teicoplanin earlier in the HD patients, if IE is serious and the antibiotic sensitivity test is not yet available.

Although the criteria for the surgical treatment of IE were the same for both HD and non-HD patients in our hospital, more non-HD patients received surgical treatment for IE than did HD patients. This was not because of the differences in disease severity between both groups but merely reflected the higher risks of operation and greater numbers of comorbidities in the HD patients, which prevented them from undergoing surgery for IE. Several reports have described a high in-hospital mortality rate among HD patients with IE. ${ }^{14,30-32}$ By contrast, the in-hospital mortality rate among non-HD patients with IE is varied across reports. ${ }^{15,33-35} \mathrm{~A}$ recent study comparing the clinical outcomes of IE in the HD and non-HD patients showed a similar in-hospital mortality rate in both the groups but a lower metastatic infection in the HD patients. ${ }^{36}$ Our study also demonstrated no difference in in-hospital deaths in the HD and non-HD patients. The high in-hospital mortality rate in both the groups in our study may be attributed to the inclusion criterion that only patients with echocardiogramconfirmed IE were enrolled. As the size of vegetations is a risk factor of mortality, ${ }^{19}$ the in-hospital mortality rate was therefore high in our study. In addition, this study analyzed the IE data of earlier years, where there was a higher in-hospital mortality rate, with those of the recent reports. ${ }^{27,37,38}$ With early surgical intervention, improvement in early diagnosis, and advancement of medical care, the in-hospital mortality rate of IE has been reduced in recent years.

This study has several limitations. First, this study sample was restricted to patients with echocardiography-confirmed IE and excluded those with possible IE without vegetations on heart valves, leading to selection bias in disease severity. Second, this study enrolled patients with IE in early years and showed a high in-hospital mortality rate. This study may not reflect a recent improvement in reducing the in-hospital mortality rate due to advancement in surgical intervention and medical care. Nevertheless, this study provides important comparisons of IE between the HD and non-HD groups.

\section{Conclusion}

This study demonstrated a greater frequency of MV involvement and S. aureus, especially MRSA, as the main causative organism in the HD patients with IE compared with the non-HD patients with IE. 


\section{Acknowledgments}

This study was supported by grants from the Chung Gang Medical Research Project to Ya-Chung Tian and ChingChung Hsiao (CORPG3F0191 and CORPG3F0221). The authors thank Shou-Hsuan Liu, Cheng-Chia Lee, Kun-Hua Tu, Chan-Yu Lin, and Ming-Yang Chang for assistance and comments that greatly improve the article.

\section{Disclosure}

The authors report no conflicts of interest in this work.

\section{References}

1. Marr KA, Kong L, Fowler VG, et al. Incidence and outcome of Staphylococcus aureus bacteremia in hemodialysis patients. Kidney Int. 1998;54(5):1684-1689.

2. Wilson WR, Karchmer AW, Dajani AS, et al. Antibiotic treatment of adults with infective endocarditis due to streptococci, enterococci, staphylococci, and HACEK microorganisms. American Heart Association. JAMA. 1995;274(21):1706-1713.

3. Small PM, Chambers HF. Vancomycin for Staphylococcus aureus endocarditis in intravenous drug users. Antimicrob Agents Chemother. 1990;34(6):1227-1231.

4. Levine DP, Fromm BS, Reddy BR. Slow response to vancomycin or vancomycin plus rifampin in methicillin-resistant Staphylococcus aureus endocarditis. Ann Intern Med. 1991;115(9):674-680.

5. Takahashi C, Warrak EA, Ruzany F. [Infectious endocarditis in patients on periodic hemodialysis]. AMB Rev Assoc Med Bras. 1991; 37(3):119-126. Portuguese [with English abstract].

6. Robinson DL, Fowler VG, Sexton DJ, Corey RG, Conlon PJ. Bacterial endocarditis in hemodialysis patients. Am J Kidney Dis. 1997;30(4): 521-524.

7. Strom BL, Abrutyn E, Berlin JA, et al. Risk factors for infective endocarditis: oral hygiene and nondental exposures. Circulation. 2000; 102(23):2842-2848.

8. Marr KA, Sexton DJ, Conlon PJ, Corey GR, Schwab SJ, Kirkland KB. Catheter-related bacteremia and outcome of attempted catheter salvage in patients undergoing hemodialysis. Ann Intern Med. 1997;127(4): 275-280.

9. Minga TE, Flanagan KH, Allon M. Clinical consequences of infected arteriovenous grafts in hemodialysis patients. Am J Kidney Dis. 2001 38(5):975-978.

10. Kessler M, Hoen B, Mayeux D, Hestin D, Fontenaille C. Bacteremia in patients on chronic hemodialysis. A multicenter prospective survey. Nephron. 1993;64(1):95-100.

11. Saad TF. Bacteremia associated with tunneled, cuffed hemodialysis catheters. Am J Kidney Dis. 1999;34(6):1114-1124.

12. Berlin JA, Abrutyn E, Strom BL, et al. Incidence of infective endocarditis in the Delaware Valley, 1988-1990. Am J Cardiol. 1995;76(12): 933-936.

13. Leonard A, Raij L, Shapiro FL. Bacterial endocarditis in regularly dialyzed patients. Kidney Int. 1973;4(6):407-422.

14. McCarthy JT, Steckelberg JM. Infective endocarditis in patients receiving long-term hemodialysis. Mayo Clin Proc. 2000;75(10): 1008-1014.

15. Nori US, Manoharan A, Thornby JI, Yee J, Parasuraman R, Ramanathan V. Mortality risk factors in chronic haemodialysis patients with infective endocarditis. Nephrol Dial Transplant. 2006;21(8):2184-2190.

16. Doulton T, Sabharwal N, Cairns HS, et al. Infective endocarditis in dialysis patients: new challenges and old. Kidney Int. 2003;64(2): 720-727.

17. Kamalakannan D, Pai RM, Johnson LB, Gardin JM, Saravolatz LD Epidemiology and clinical outcomes of infective endocarditis in hemodialysis patients. Ann Thorac Surg. 2007;83(6):2081-2086.
18. Fukuchi T, Iwata K, Ohji G. Failure of early diagnosis of infective endocarditis in Japan-a retrospective descriptive analysis. Medicine (Baltimore). 2014;93(27):e237.

19. Rohmann S, Erbel R, Görge G, et al. Clinical relevance of vegetation localization by transoesophageal echocardiography in infective endocarditis. Eur Heart J. 1992;13(4):446-452.

20. Jones DA, McGill LA, Rathod KS, et al. Characteristics and outcomes of dialysis patients with infective endocarditis. Nephron Clin Pract. 2013;123(3-4):151-156.

21. Chou MT, Wang JJ, Wu WS, et al. Epidemiologic features and longterm outcome of dialysis patients with infective endocarditis in Taiwan. Int J Cardiol. 2015;179:465-469.

22. Sox HC Jr, Liang MH. The erythrocyte sedimentation rate. Guidelines for rational use. Ann Intern Med. 1986;104(4):515-523.

23. Allin KH, Nordestgaard BG. Elevated C-reactive protein in the diagnosis, prognosis, and cause of cancer. Crit Rev Clin Lab Sci. 2011;48(4):155-170.

24. Hogevik H, Olaison L, Andersson R, Alestig K. C-reactive protein is more sensitive than erythrocyte sedimentation rate for diagnosis of infective endocarditis. Infection. 1997;25(2):82-85.

25. Brouillard M, Reade R, Boulanger E, et al. Erythrocyte sedimentation rate, an underestimated tool in chronic renal failure. Nephrol Dial Transplant. 1996;11(11):2244-2247.

26. Olaison L, Hogevik H, Alestig K. Fever, C-reactive protein, and other acute-phase reactants during treatment of infective endocarditis. Arch Intern Med. 1997;157(8):885-892.

27. Vasudev R, Shah P, Kaur S, et al. Infective endocarditis in hemodialysis patients. J Heart Valve Dis. 2016;25(3):369-374.

28. Wang A, Athan E, Pappas PA, et al; International Collaboration on Endocarditis-Prospective Cohort Study Investigators. Contemporary clinical profile and outcome of prosthetic valve endocarditis. JAMA. 2007;297(12):1354-1361

29. Benito N, Miró JM, de Lazzari E, et al; ICE-PCS (International Collaboration on Endocarditis Prospective Cohort Study) Investigators. Health care-associated native valve endocarditis: importance of nonnosocomial acquisition. Ann Intern Med. 2009;150(9):586-594.

30. Chang CF, Kuo BI, Chen TL, Yang WC, Lee SD, Lin CC. Infective endocarditis in maintenance hemodialysis patients: fifteen years' experience in one medical center. J Nephrol. 2004;17(2):228-235.

31. Maraj S, Jacobs LE, Kung SC, et al. Epidemiology and outcome of infective endocarditis in hemodialysis patients. Am J Med Sci. 2002; 324(5):254-260.

32. Rekik S, Trabelsi I, Hentati M, et al. Infective endocarditis in hemodialysis patients: clinical features, echocardiographic data and outcome: a 10-year descriptive analysis. Clin Exp Nephrol. 2009;13(4): $350-354$.

33. Thalme A, Westling K, Julander I. In-hospital and long-term mortality in infective endocarditis in injecting drug users compared to nondrug users: a retrospective study of 192 episodes. Scand J Infect Dis. 2007;39(3):197-204.

34. Smith JM, So RR, Engel AM. Clinical predictors of mortality from infective endocarditis. Int J Surg. 2007;5(1):31-34.

35. Hill EE, Herijgers P, Claus P, Vanderschueren S, Herregods MC, Peetermans WE. Infective endocarditis: changing epidemiology and predictors of 6-month mortality: a prospective cohort study. Eur Heart J. 2007;28(2):196-203.

36. Abu Sitta E, Habte-Gabr E, Qaraghan Z, Aljariri Alhesan N, RiosBedoya $\mathrm{C}$. Infective endocarditis in haemodialysis patients: lower complications and same mortality rate as in non-haemodialysis patients Infect Dis (Lond). 2017;49(4):308-311.

37. Ludvigsen LU, Dalgaard LS, Wiggers H, et al. Infective endocarditis in patients receiving chronic hemodialysis: a 21-year observational cohort study in Denmark. Am Heart J. 2016;182:36-43.

38. Durante-Mangoni E, Pafundi PC, Ravasio V, et al. Current features of infective endocarditis in persons on hemodialysis: a prevalence study with case control design from the prospective multicenter SEI cohort. Infection. 2016;44(4):467-474. 
Therapeutics and Clinical Risk Management

Dovepress

\section{Publish your work in this journal}

Therapeutics and Clinical Risk Management is an international, peerreviewed journal of clinical therapeutics and risk management, focusing on concise rapid reporting of clinical studies in all therapeutic areas, outcomes, safety, and programs for the effective, safe, and sustained use of medicines. This journal is indexed on PubMed Central, CAS,

EMBase, Scopus and the Elsevier Bibliographic databases. The manuscript management system is completely online and includes a very quick and fair peer-review system, which is all easy to use. Visit $\mathrm{http}: / / \mathrm{www}$.dovepress.com/testimonials.php to read real quotes from published authors.

Submit your manuscript here: http://www.dovepress.com/therapeutics-and-clinical-risk-management-journal 